\title{
Intégration des transports et de l'aménagement du territoire au niveau métropolitain à Toronto et à Chicago : perspectives de gouvernance verticale et horizontale Integrating transportation and land use at the metropolitan level in Toronto and Chicago: Perspectives of vertical and horizontal governance Integración del transporte y de la planificación del territorio a nivel metropolitano de Toronto y de Chicago: Perspectivas de gobernanza vertical y horizontal
}

\author{
Fanny R. Tremblay-Racicot et Jean Mercier
}

Volume 58, numéro 164, septembre 2014

Les paradoxes et défis actuels du développement urbain et métropolitain

Version originale soumise en mars 2013. Version révisée reçue en janvier 2015.

URI : https://id.erudit.org/iderudit/1031167ar

DOI : https://doi.org/10.7202/1031167ar

\section{Aller au sommaire du numéro}

Éditeur(s)

Département de géographie de l’Université Laval

ISSN

0007-9766 (imprimé)

1708-8968 (numérique)

Découvrir la revue

Citer cet article

Tremblay-Racicot, F. R. \& Mercier, J. (2014). Intégration des transports et de l'aménagement du territoire au niveau métropolitain à Toronto et à Chicago : perspectives de gouvernance verticale et horizontale. Cahiers de géographie du Québec, 58(164), 213-232. https://doi.org/10.7202/1031167ar

\section{Résumé de l'article}

Cet article porte sur l'intégration de l'aménagement du territoire et des transports au niveau métropolitain en Amérique du Nord. Au moyen d'une analyse de contenu d'entretiens semi-dirigés et d'une recherche documentaire, deux grandes régions métropolitaines, Toronto et Chicago, sont comparées quant à leur cheminement institutionnel vers une plus grande intégration de la planification des transports et de l'aménagement du territoire. Les interventions des différents paliers d'autorité sont décrites et comparées, mettant en exergue la manière dont le défi d'intégration est entrepris des deux côtés de la frontière. Alors que le gouvernement de l'Ontario a emprunté une approche législative et davantage centralisée, avec la création d'une agence de transport métropolitaine contrôlée par le ministre des Transports et l'adoption de deux lois de gestion de la croissance avec obligation de conformité, le gouvernement de l'Illinois a opté pour une approche plus décentralisée, avec la création d'une agence de planification régionale aux capacités de financement et à l'autorité limitées, dont le fonctionnement est basé sur la coopération volontaire entre acteurs locaux. Les différentes trajectoires empruntées dans les deux régions indiquent que la dimension verticale de la gouvernance constitue un passage obligé, à certaines étapes des politiques visant l'intégration des transports et de l'aménagement du territoire au niveau régional, et soulève des questions quant à l'impact du design institutionnel des nouvelles agences de planification sur le financement des projets de transport et les décisions relatives à l'utilisation du sol. 


\title{
Intégration des transports et de l'aménagement du territoire au niveau métropolitain à Toronto et à Chicago: perspectives de gouvernance verticale et horizontale
}

\author{
Integrating transportation and land use \\ at the metropolitan level in Toronto and \\ Chicago: Perspectives of vertical and \\ horizontal governance \\ Integración del transporte y de la planificación \\ del territorio a nivel metropolitano de Toronto \\ y de Chicago: Perspectivas de gobernanza \\ vertical y horizontal
}

\author{
Fanny R. TREMBLAY-RACICOT \\ Department of Geography and Urban Studies \\ Temple University, Philadelphie \\ Ftracicot@hotmail.com \\ Jean MERCIER \\ Département de science politique \\ Université Laval \\ Jean.Mercier@pol.ulaval.ca
}

\section{Résumé}

Cet article porte sur l'intégration de l'aménagement du territoire et des transports au niveau métropolitain en Amérique du Nord. Au moyen d'une analyse de contenu d'entretiens semidirigés et d'une recherche documentaire, deux grandes régions métropolitaines, Toronto et Chicago, sont comparées quant à leur cheminement institutionnel vers une plus grande intégration de la planification des transports et de l'aménagement du territoire. Les interventions des différents paliers d'autorité sont décrites et comparées, mettant en exergue la manière dont le défi d'intégration est entrepris des deux côtés de la frontière. Alors que le gouvernement de l'Ontario a emprunté une approche législative et davantage centralisée, avec la création d'une agence de transport métropolitaine contrôlée par le ministre des Transports et l'adoption de deux lois de gestion de la croissance avec obligation de conformité, le gouvernement de l'Illinois a opté pour une approche plus décentralisée, avec la création d’une agence de planification régionale aux capacités de financement et à l'autorité limitées, dont le fonctionnement est basé sur la coopération volontaire entre acteurs locaux. Les différentes trajectoires empruntées dans les deux régions indiquent que la dimension verticale de la gouvernance constitue un passage obligé, à certaines étapes des politiques visant l'intégration des transports et de l'aménagement du territoire au niveau régional, et soulève des questions quant à l'impact du design institutionnel des nouvelles agences de planification sur le financement des projets de transport et les décisions relatives à l'utilisation du sol.

\section{Mots-clés}

Transport, aménagement du territoire, planification intégrée, stratégie de gestion de la croissance, gouvernance urbaine, gouvernance multiniveau, design institutionnel, Canada, États-Unis, étude comparative. 


\begin{abstract}
This article focuses on the integration of transportation and land use planning at the metropolitan level in North America. Using semi-structured interviews and documentary sources, two large metropolitan areas, Toronto and Chicago, are compared in terms of their institutional evolution towards the goal of integrating regional transportation and land use planning. Public policies of different levels of government are documented, outlining the differences in responding to the challenges of planning integration in each city. In Toronto, a regional transportation agency controlled by the ministry of Transportation was created, and two pieces of legislation implementing a growth management strategy were adopted. In Chicago, a regional planning agency with the mandate of integrating transportation and land use planning was created, but its funding capacity and authority in terms of land use planning are limited, and thus more dependent on voluntary cooperation with the numerous local entities. These two different trajectories of reform indicate that the vertical dimension of governance constitutes an obligatory step on the path to the integration of transportation and land use planning at the regional level, and raise some questions around the impacts on transportation funding and land use decisions of the institutional design of new regional planning agencies.
\end{abstract}

\title{
Keywords
}

Transport, land use, integrated planning, growth management strategy, urban governance, multi-level governance, institutional design, Canada, United States, comparative study.

\section{Resumen}

Este artículo trata sobre la integración de la planificación del territorio y del transporte a nivel metropolitano en América del Norte. Gracias al análisis de contenido de entrevistas casi dirigidas y de documentos, se comparan dos grandes regiones metropolitanas: Toronto y Chicago, en cuanto al proceso institucional hacia una mayor integración entre la planificación del transporte y la territorial. Se describen intervenciones a diferentes grados de autoridad y se las compara, destacando la manera cómo el desafío de integración se enfrenta en los dos lados fronterizos. Mientras que el gobierno de Ontario ha adoptado un enfoque legislativo y más centralizado, creando un agencia metropolitana de transportes controlada por el Ministerio de Transportes y por dos leyes de gestión de desarrollo con obligación de conformidad, el gobierno de Illinois ha optado por un enfoque más descentralizado, creando un agencia de planificación regional, con posibilidades de financiamiento y autoridad limitada, cuyo funcionamiento se basa en la cooperación voluntaria de los actores locales. Las trayectorias diferentes de las dos regiones indican que la dimensión vertical de gobernanza es obligatoria en ciertas etapas de la política que tienden a integrar los transporte y la planificación del territorio a nivel regional. Esas trayectorias interrogan el impacto del design institucional de las nuevas agencias de panificación sobre el financiamiento de proyectos de transporte y las decisiones relativas a la utilización del suelo.

\section{Palabras claves}

Transporte, planificación territorial, planificación integrada, estrategia de gestión del desarrollo, gobernanza urbana, gobernanza a varios niveles, creación institucional, Canadá, Estados Unidos, estudio comparativo. 


\section{Contexte}

L'intégration de la planification des transports et l'aménagement du territoire à l'échelle régionale est un objectif relativement récent lié à un changement de paradigme dans le domaine des transports urbains, lequel reconnaît l'impact de l'utilisation du territoire sur les habitudes de transport (Litman, 2013). La complexité du problème de l'intégration de la planification a suscité l'attention des chercheurs, des militants et des gouvernements, qui ont récemment tenté diverses approches et réformes afin de simplifier le processus décisionnel historiquement cloisonné et fragmenté entre différents ordres de gouvernement et autres organisations privées ou parapubliques, et ce, de la planification à la mise en œuvre, en passant par le financement des plans et projets de transport et d'aménagement. Alors que certaines réformes des structures de gouvernance font appel à des politiques incitatives basées sur la collaboration volontaire et le consensus, relativement décentralisées, d'autres sont davantage fondées sur la législation et les processus de conformité, plus centralisés (Weir, 2000; Alexander, 2010). Bien qu'il ne faille pas réduire les réformes institutionnelles intégrant transports et aménagement du territoire à cette dichotomie (décentralisation/centralisation), celle-ci nous paraît importante dans la structuration du processus décisionnel du défi qui se trouve devant nous. Parce que les régions métropolitaines impliquent aujourd'hui, et de plus en plus, à la fois plusieurs niveaux de gouvernement ainsi que plusieurs intervenants non gouvernementaux, on peut dire que nous nous situons dans un cadre de gouvernance multiniveau, où les villes sont un de ces «sites of governance» (Horak et Young, 2012; Divay et Paquin, 2013: 8), un lieu privilégié pour étudier la gouvernance multiniveau.

D’une façon générale, le thème de la gouvernance est associé à des processus de décision interactifs et horizontaux. Ce regard porté sur la gouvernance, de descriptif qu'il était au départ, a souvent pris une allure normative et prescriptive, au point où l'on a supposé que les décisions prises au niveau local étaient de meilleure qualité que celles prises au niveau central (Young, 2012: 10) et que, finalement, «networked governance can be more efficient and flexible than traditional bureaucratic administration» (Young, 2012: 6, reprenant les propos de Rhodes, 1996). Par contre, nous sommes d'avis que, même dans un cadre de gouvernance, et plus particulièrement dans un cadre de gouvernance multiniveau, nous ne pouvons pas évacuer complètement le rôle des autorités publiques, dont l'intervention peut se traduire, dans certaines circonstances, en un mode centralisé, voire top down et coercitif.

Deux raisons nous poussent à tenir compte de cette dimension. La première vient du fait que l'intégration entre les dimensions transport et aménagement constitue un défi important en matière de coordination. Bien sûr, il existe en théories administratives plusieurs façons de coordonner des tâches. La coordination par la centralisation et les directives de type top-down font partie de cette liste de modalités de coordination. En particulier, l'objectif d'agir sur la demande en transport (et non plus seulement sur l'offre) (Litman, 2013: 26), celui d'une vision plus intégrée de la ville, ainsi que l'objectif de transformer les infrastructures routières en nouveaux «architectes de l'espace public» (Bourdages et Champagne, 2012: 10-16) ne nous apparaissent pas comme devant exclure l'option d'une approche plus centralisée de la prise de décision. 
Notre deuxième raison vient du fait que, même si «souligner l'importance de l'administration peut sembler un truisme» (Divay et Paquin, 2013: 11), il faut mentionner que l'administration publique est un acteur «qui occupe une place particulière en raison de son importance et de son statut» (Ibid.) et que «les dispositifs de la gouvernance multiniveau sont surtout portés par l'administration publique et pourtant son travail est peu décortiqué» (Ibid. : 19). Or, un des modes de prise de décision de l'administration publique peut être, par définition, centralisé et hiérarchique.

Au contraire de Klijn et Koppenjan (2012), nous ne considérons pas une approche hiérarchique comme étant un piège, mais plutôt comme une des options qui se présentent lorsqu'il y a un défi de coordination. Le mode de gouvernance, au sens où plusieurs partenaires interagissent sans cadre hiérarchique, ne doit pas non plus être considéré, au départ et sans soutien empirique, comme supérieur pour tous les défis de politique publique. Robert Young nous propose d'ailleurs une certaine prudence à cet égard (2012: 18-19), une prudence réitérée par Martin Horak (2012b : 366-367) en ce qui a trait aux études sur les grandes villes canadiennes.

Cette recherche empirique au sujet des transformations institutionnelles visant à intégrer les dimensions transport et aménagement dans les territoires métropolitains est d'autant plus justifiée par le fait qu'on n'en sait que très peu à propos de ces nouvelles initiatives et de leur impact réel sur l'aménagement du territoire et la planification des transports (Margerum et al., 2011). Tout en s'intéressant aux dimensions géographiques du lien transport-aménagement, nous nous attardons donc surtout à ce qui est en amont de ce lien, à savoir les dynamiques institutionnelles du processus de prise de décision, et la manière dont celles-ci pourraient influencer les dimensions géographiques et urbaines à plus long terme. Le caractère récent de la problématique d’intégration et des changements de structure de gouvernance prescrit que nous nous attardions d'abord à comprendre la manière dont les nouvelles institutions modifient la dynamique de pouvoirs entre acteurs, laquelle déterminera l'étendue probable des changements en ce qui a trait aux types de décisions qui sont prises dans les diverses instances.

Cette étude contribue donc à explorer et à documenter la manière dont certaines réformes institutionnelles peuvent être vectrices de changement ou, au contraire, constituent un frein à l'intégration de la planification des transports et de l'aménagement du territoire à l'échelle métropolitaine. Plus précisément, elle a pour objet les politiques et les processus par lesquels le transport et l'aménagement du territoire sont intégrés dans les régions de Toronto (Canada) et Chicago (États-Unis), lesquelles présentent un profil similaire en termes de géographie et de démographie, mais où le gouvernement provincial et celui de l'État ont récemment adopté une structure de gouvernance très différente, voire opposée, pour la gestion de la planification des transports et de l'aménagement du territoire. Alors que le gouvernement de l'Ontario a emprunté une approche législative et davantage centralisée, avec la création d'une agence de transport métropolitaine (Metrolinx) contrôlée par le ministre des Transports, ainsi qu'avec l'adoption de deux lois de gestion de la croissance, le gouvernement de l'Illinois a emprunté une approche plus décentralisée, avec la création d'une agence de planification des transports et d'aménagement du territoire (CMAP) dont le fonctionnement dépend davantage de la bonne volonté des acteurs locaux. 
À partir de 48 entretiens semi-dirigés ${ }^{1}$, ainsi que de ressources documentaires, nous décrivons l'éventail des politiques et des pratiques institutionnelles des autorités gouvernementales et quasi gouvernementales, de façon à lever le voile sur la manière dont le défi de l'intégration de l'aménagement du territoire et des transports est relevé dans un contexte de fragmentation institutionnelle au niveau régional à Toronto et à Chicago. Bien que l'étude originale dont cette analyse provient ait un objectif plus large (celui d'identifier le choix et la séquence des instruments de politique publique pour réduire les émissions de gaz à effet de serre provenant des transports urbains dans cinq régions métropolitaines des Amériques), nous nous concentrons ici uniquement sur les politiques, plans et projets intégrant les transports et l'aménagement du territoire, plus particulièrement sur les aspects institutionnels et ceux de la prise de décision, tel que mentionné plus tôt. Ainsi, la présente recherche emprunte une démarche d'exploration des politiques et des processus de prise de décision par lesquels l'aménagement du territoire et la planification des transports sont intégrés au niveau métropolitain, en tentant de comprendre comment les relations verticales et horizontales entre différentes organisations peuvent influencer la planification métropolitaine de l'aménagement du territoire et des transports aux niveaux local et métropolitain.

La première section dresse un portrait succinct de la littérature relative à l'intégration de l'aménagement du territoire et des transports, ainsi qu'à la question institutionnelle au niveau métropolitain. À la revue de la littérature succède une description de la méthode utilisée, incluant une présentation des régions métropolitaines de Toronto et Chicago. Les résultats sont par la suite présentés par ville et par palier de gouvernement. Finalement, cet article se conclut par une discussion sur l'implication et la portée théorique des résultats.

\section{Intégration de l'aménagement du territoire et des transports}

L'interaction et l'interdépendance entre les transports et l'aménagement du territoire, ainsi que l'efficacité des différents outils de politique publique sur les habitudes de transport et le choix de location des résidences et des entreprises, sont des éléments difficiles à mesurer et font l'objet de vifs débats scientifiques (Giuliano, 1995; Handy et al., 2005; Neuman, 2005 ; Cervero et Duncan, 2006; Forsyth et al., 2007; Transportation Research Board, 2009). Cependant, la nécessité de coordonner la planification de l'aménagement du territoire et des transports fait davantage consensus. En effet, un nouveau paradigme en planification des transports, parfois appelé «mobilité durable» (Bourdages et Champagne, 2012) mais aussi «accessibilité» (Litman, 2013), change la manière dont est définie la problématique des transports et les solutions à y apporter, plaçant à l'avant-plan les outils de gestion de la demande des transports, comme l'intégration de la planification des transports et de l'aménagement du territoire (Ibid.). L’ancien paradigme évaluait la performance des systèmes de transport selon des critères de vitesse, de coût, et de caractère pratique des déplacements motorisés, favorisant ainsi les améliorations orientées vers les transports en automobile, alors

1 Le projet de recherche global comprend cinq villes (Seattle, Chicago, Toronto, Montréal et Curitiba), pour un total de 60 entrevues. Toutes les entrevues des régions étasuniennes et canadiennes ont été mises à contribution pour la rédaction de cet article, puisque les réponses ayant trait aux relations intergouvernementales dans un système fédéral peuvent parfois s'appliquer à toutes les régions d'un même État national. Ainsi, les entrevues de Seattle, Chicago, Toronto et Montréal ont été numérotées de 1 à 48 . Cependant, seules les entrevues d'où ont été tirées des informations spécifiques sont citées. 
que le nouveau paradigme, basé sur l'accessibilité aux services et aux activités, considère un éventail plus large de modes, d'objectifs, d'impacts et de possibilités d'amélioration d'infrastructures de transport (Johnston, 2004; Litman, 2013). Ainsi, de nouvelles stratégies visant l'accessibilité, la diminution de l'étalement urbain et l'amélioration de la qualité de l'air sont mises de l'avant, comme celles d'augmenter la densité de population et la mixité des fonctions, d'augmenter ou de bonifier l'offre de service des transports collectifs, et de diriger la croissance démographique vers des milieux bâtis en redéveloppant certains secteurs où les services de transport en commun sont déjà existants (Johnston, 2004: 112; Black, 2010 : 139).

L'intégration de l'aménagement du territoire et des transports est désormais un objectif de politique publique dans un grand nombre de juridictions. Aux États-Unis, le passage de l'Intermodal Surface Transportation Efficiency Act (ISTEA), en 1991, du Transportation Equity Act for the 21 ${ }^{\text {st }}$ Century (TEA-21), en 1998, et du Safe, Accountable, Efficient, Transportation Equity Act: A Legacy for Users (SAFETEA-LU), en 2005, ont contribué à la redéfinition du paradigme au niveau fédéral américain en élargissant l'étendue des programmes de financement pour inclure les transports collectifs, les modes de transport alternatif, l'aménagement du territoire, ainsi que d'autres objectifs régionaux allant au-delà de l'accroissement quantitatif de la mobilité, comme la responsabilité sociale, économique et environnementale (Wheeler, 2002; Hamilton et al., 2008; Weir et al., 2009). Cependant, on n'en sait que très peu sur la manière dont se traduisent ces objectifs dans les politiques et pratiques des secteurs publics et parapublics américains au niveau régional et local, sinon que la mise en œuvre des lois et programmes fédéraux étasuniens rencontre des obstacles importants, notamment la résistance des gouvernements locaux et étatiques à toute dévolution de pouvoir au niveau régional et les capacités limitées des agences métropolitaines de planification qui en résultent (Hamilton et al., 2008; Weir et al., 2009). La valorisation du home rule, un principe qui favorise une décentralisation poussée de la prise de décision, composante essentielle de «l'exception américaine» en administration publique (Riggs, 1998; Hibbing et Thiesse-Moss, 2001), expliquerait en partie cette résistance des pouvoirs locaux.

\section{La question institutionnelle au niveau métropolitain}

La source du débat actuel entourant la fragmentation institutionnelle au niveau métropolitain remonte aux années 1950, à l'époque du boom de l'après-Deuxième Guerre mondiale qui engendra une croissance rapide des banlieues. Le débat opposa les partisans de la réforme métropolitaine, ou des fusions municipales, souvent associée à des processus centralisés, aux partisans de l'école des choix publics, ou de la fragmentation institutionnelle, souvent associée à des processus décentralisés, et ce, jusqu'à l'émergence du nouveau régionalisme, dans les années 1990. Les prochains paragraphes traitent des plus récentes avenues de recherche sur la question métropolitaine.

Le nouveau régionalisme, un ensemble de points de vue mettant l'accent sur le besoin d'intégrer l'aménagement physique, le design urbain et la planification équitable en réponse aux problèmes urbains contemporains comme l'étalement urbain, les iniquités, la congestion et la détérioration de l'environnement, met de l'avant la coopération régionale comme vecteur de changement (Wheeler, 2002). Ainsi, les mouvements tels 
que l'«urbanisme nouveau», la croissance intelligente et le développement durable suggèrent de modifier la forme urbaine, et non pas la structure politique des régions métropolitaines (Calthorpe et Fulton, 2001). Autrement dit, ils préconisent l'intégration des transports et l'aménagement du territoire, mais ne proposent pas de modus operandi au niveau institutionnel ou politique, mis à part la collaboration d'acteurs locaux. Cette «non-décision» sur le plan du choix institutionnel nous renvoie à un processus de gouvernance, dans le sens d'une collaboration horizontale entre partenaires interdépendants, mais non regroupés dans un ordre hiérarchique. Aussi incluses sous le parapluie du nouveau régionalisme sont les tentatives de gestion de la croissance de deuxième et troisième générations, qui furent d'abord développées dans les années 1970 en Oregon et adaptées en Floride, dans le Maine, le Maryland, le New Jersey, le Rhode Island, le Vermont et l'État de Washington (Porter, 1992; Deal et al., 2009). Ces politiques de gestion de la croissance, adoptées par les gouvernements de l'État, visent à diriger la croissance dans certaines zones au niveau régional et sont mises en œuvre par les gouvernements municipaux qui doivent généralement s'y conformer à travers leur plan d'aménagement. Les études empiriques démontrent qu'elles peuvent contribuer à un accroissement des usagers du transport en commun en limitant l'exode des quartiers centraux (Deal et al., 2009: 21). Cependant, parce que les politiques de gestion de la croissance et la mise en place de gouvernements régionaux requièrent une forte coercition, elles ne sont pas perçues comme toujours réalistes sur le plan politique. C'est pourquoi les commentateurs de la scène métropolitaine tendent à mettre de l'avant des solutions basées sur un développement graduel du capital social, sur des initiatives de collaboration sectorielle ad hoc, ainsi que sur des cadres d'incitatifs et de mandats entre les différents paliers de gouvernement, malgré toutes les limites qu'une approche volontaire peut comporter (Brenner, 2002: 9; Wheeler, 2002: 267-270; Alexander, 2010: 643 ; Nelles, 2012: 5). Autrement dit, on est renvoyé, ici aussi, à une forme de gouvernance décentralisée, avec ses limites inhérentes, d'autant plus que, dans le cas de la planification régionale des transports et de l'aménagement du territoire, on doit s'entendre sur des zones géographiques qui seront favorisées en termes d'investissements et d'infrastructures, et d'autres qui le seront moins.

Par ailleurs, dans la pratique, plusieurs réformes ont été mises en application, dont les fusions municipales (villes souvent centralisées à la fois dans leur création et leur mode de fonctionnement) et la création de gouvernements locaux correspondant aux frontières des régions métropolitaines, mais aussi la création d'autorités de diverses natures (Norton, 1994: 110-112). Règle générale, l’Europe a opté pour les fusions municipales et l'intégration des structures et des services, alors que les ÉtatsUnis tendent vers les autorités à mandat spécifique qui dépendent de la coopération volontaire (Ibid. : 393). On peut penser que cette différence relève des processus plus hiérarchiques et top down en Europe. On peut aussi penser que les villes canadiennes se trouveraient, comme c'est souvent le cas en administration publique, quelque part entre les modèles européen et étasunien.

La multiplication des autorités à mandat spécifique, aux États-Unis, n’est pas sans exacerber le phénomène de fragmentation institutionnelle, au point où l'enchevêtrement des frontières entre les autorités de services publics fut décrit comme étant un «chaos organisé» (Ibid. : 44). Face à cet état de fait, la littérature scientifique se situe désormais, en termes de gouvernance multiniveau (ou gouvernance à paliers multiples) et de collaboration horizontale et verticale, entre les différents 
ordres de gouvernement et autres organisations parapubliques, la société et le marché (Brenner, 2002; Wheeler, 2002; Weir et al., 2009; Alexander, 2011 ; Horak et Young, 2012; Nelles, 2013). Certains auteurs soulignent les limites du processus de collaboration délibérative pour la planification régionale et la nécessité des réformes de l'aménagement du territoire centralisées et mandatées par l’État (Weir, 2000; Alexander, 2010). Une autre direction de la recherche consiste à évaluer la force des partenariats horizontaux au niveau régional et la façon dont ils peuvent jouer dans les arènes politiques des niveaux supérieurs de gouvernement (Weir et al., 2009; Nelles, 2013). L'approche de la gouvernance urbaine, quant à elle, souligne l'importance des nouvelles structures institutionnelles liant le gouvernement, la société et le marché, qui sont plus complexes et plus difficiles à gérer que les institutions gouvernementales centralisées conventionnelles, mais qui ont un rôle crucial pour naviguer dans les eaux des environnements urbains contemporains (Pierre et Peters, 2012: 83).

La présence de différents patrons de gouvernance dans les défis de la gouvernance multiniveau nous amène à nous interroger sur la place que celle-ci peut occuper à différentes étapes du processus décisionnel. Si elle est sans doute indispensable à l'étape de la consultation, son rôle est moins clair aux étapes ultérieures. Selon Horak, elle serait plus facile à exercer lorsque la fragmentation du pouvoir et des agendas est moins grande, c'est-à-dire quand elle serait moins nécessaire, ce qui nous amène à douter de l'affirmation selon laquelle la gouvernance multiniveau serait supérieure à l'exercice de l'autorité par un niveau de gouvernement (Horak, 2012a: 256).

Par notre étude, nous voulons contribuer au débat entre les approches législative, hiérarchique et top down, d'une part, et les approches coopérative et horizontale, d'autre part, en explorant la manière dont l'intégration de la planification des transports et de l'aménagement du territoire s'effectue aux niveaux local et métropolitain dans deux régions qui furent récemment le théâtre de réformes structurelles paraissant, au premier regard, de nature opposée.

\section{Méthodologie et stratégie de recherche}

Cette recherche est basée sur une étude de cas comparée des régions métropolitaines de Toronto, située dans la province de l'Ontario, au Canada, et de Chicago, située dans l'État de l'Illinois, aux États-Unis. Le choix de cette paire a été effectué sur la base de la similitude des deux villes en termes de géographie, de démographie et de l'importance qu'elles ont en matière de transport pour leur région et pays respectifs, mais aussi sur la base du fait que leur structure de gouvernance régionale fut récemment l'objet d'une réforme. Nous présentons ainsi deux cas ayant chacun son nouveau modèle de gouvernance à première vue opposé à l'autre (Toronto étant, entre autres, plutôt centralisé et Chicago, plutôt décentralisé), en décrivant les politiques, les processus décisionnels et les pratiques institutionnelles qui en découlent en matière de transport et d'aménagement (Yin, 2003 ; Flyvbjerg, 2006). En comparant Toronto et Chicago, nous pensons respecter l'idée que, pour réellement comparer deux entités sur une dimension particulière, il importe de tenir constants (et semblables) d'autres éléments. Dans le cas qui nous occupe ici, les éléments autres qu'institutionnels nous sont apparus comme remarquablement semblables, comme le démontrent les données du tableau 1. Bien entendu, d'autres critères ont influencé le choix de cette paire, 
notamment celui utilisé pour la sélection des cas du projet de recherche plus large à laquelle cette étude est rattachée, soit d'avoir affaire à des villes qui jouissent de parts modales en faveur des transports collectifs supérieures aux moyennes des villes nord-américaines (Freemark, 2009; Data Management Group, 2011).

Nous voulons identifier, en amont, les fondements institutionnels de l'intégration entre transports et aménagement du territoire en examinant les processus de prise de décision qui les caractérisent. Ce faisant, nous pensons contribuer à la documentation et à la compréhension des processus horizontaux et verticaux faisant partie de la gouvernance. Dans cette étude, une approche comparative est adoptée.

\section{Tableau 1 Toronto et Chicago en chiffres}

\begin{tabular}{lcc|cc}
\hline & \multicolumn{2}{c|}{ Toronto } & \multicolumn{2}{c}{ Chicago } \\
& $\begin{array}{c}\text { Toronto } \\
\text { ville }\end{array}$ & $\begin{array}{c}\text { Toronto - } \\
\text { RGTH }\end{array}$ & $\begin{array}{c}\text { Chicago - } \\
\text { ville }\end{array}$ & $\begin{array}{c}\text { Chicago - } \\
\text { métro }\end{array}$ \\
\hline Superficie $^{1}$ & $630 \mathrm{~km}^{2}$ & $8262 \mathrm{~km}^{2}$ & $606 \mathrm{~km}^{2}$ & $10544 \mathrm{~km}^{2}$ \\
Population $^{2}$ & 2615060 & 6574140 & 2714856 & 8638474 \\
Densité de population $^{3}$ & $4149 / \mathrm{km}^{2}$ & $796 / \mathrm{km}^{2}$ & $4480 / \mathrm{km}^{2}$ & $819 / \mathrm{km}^{2}$ \\
Pop. ville/Pop. métro & $40 \%$ & & $31 \%$ & \\
Nombre de municipalités $^{4}$ & 26 & & 284 & \\
Nombre de municipalités $_{\text {régionales (Ont.) ou comtés (Ill.) }}{ }^{5}$ & 4 & & 7 & \\
\hline
\end{tabular}

Note: Les données pour les régions métropolitaines se rapportent au territoire couvert par les agences régionales de planification des deux régions, soit Metrolinx pour la région du Grand Toronto et Hamilton (RGTH), et CMAP pour la région de Chicago.

Sources:

1 Toronto: Statistique Canada. 2012. Toronto, Ontario (Code 3520) and Ontario (Code 35) (table). Census Profile. 2011 Census. Statistics Canada Catalogue no. 98-316-XWE. Ottawa. Released October 24, 2012 [En ligne]. http://www12.statcan.gc.ca/census-recensement/2011/dp-pd/prof/index. cfm?Lang=E; Chicago - Métro: CMAP. 2005. Land Use Inventory [En ligne]. http://www.cmap.illinois.gov/land-use-inventory

2 Toronto en 2011: Statistique Canada. 2012. Toronto, Ontario (Code 3520) and Ontario (Code 35) (table). Census Profile. 2011 Census. Statistics Canada Catalogue no. 98-316-XWE. Ottawa. Released October 24, 2012 [En ligne]. http://www12.statcan.gc.ca/census-recensement/2011/dp-pd/prof/ index.cfm?Lang=E; Chicago - Ville en 2010: “U.S. Census Bureau Delivers Illinois' 2010 Census Population Totals, Including First Look at Race and Hispanic Origin Data for Legislative Redistricting". U.S. Census Bureau. Chicago - Métro en 2010: CMAP [En ligne]. http://www.cmap.illinois.gov/ population-forecast

3 La formule Population/Superficie est calculée pour fins de comparaison seulement.

4 Toronto: Régie des transports de la région du Grand Toronto. 2008. Le Grand Projet [En ligne]. http://www.metrolinx.com/thebigmove/Docs/ big_move/081059_MetroLinx_TheBigMoveFR_V3_SM.pdf; Chicago: CMAP. 2005. About [En ligne]. http://www.cmap.illinois.gov/about

5 Toronto: Régie des transports de la région du Grand Toronto. 2008. Le Grand Projet [En ligne]. http://www.metrolinx.com/thebigmove/Docs/ big_move/081059_MetroLinx_TheBigMoveFR_V3_SM.pdf; Chicago: CMAP. 2005. About [En ligne]. http://www.cmap.illinois.gov/about

Les données proviennent d'une douzaine d'entretiens semi-dirigés réalisés dans chaque région en 2011, soit environ cinq ans après l'adoption des réformes institutionnelles à Toronto et à Chicago. Les informateurs-clés représentent divers secteurs gouvernementaux et non gouvernementaux liés aux transports et à l'aménagement du territoire (fédéral, province/État, métropolitain, municipal, agences de planification, agences de transport et groupes d'intérêt). Aux fins de la présente étude, seules les réponses aux questions liées aux transports et à l'aménagement du territoire, au processus décisionnel et aux relations intergouvernementales ont été sélectionnées parmi toutes les réponses au questionnaire, lequel portait sur plusieurs aspects des transports, incluant l'environnement physique, social, institutionnel et 
les instruments de politique publique. Nous avons adopté une approche inductive et exploratoire, en demandant aux répondants d'identifier les politiques et projets intégrant l'aménagement du territoire et les transports, puis en leur demandant de décrire les relations intergouvernementales et le processus décisionnel ayant mené à l'adoption et à la mise en œuvre de ces politiques.

\section{Présentation des cas}

Les deux villes sont situées sur les rives d'un Grand Lac: Chicago, bordant le lac Michigan, et Toronto, bordant le lac Ontario. Si la présence du lac limite leur développement, la topographie plate du territoire sur lequel elles se trouvent est propice à l'étalement urbain, ainsi que le démontrent les données du tableau 1.

La région métropolitaine de Toronto, la plus grande région urbaine du Canada, s'étend sur toute la rive est du lac Ontario en prenant la forme d'un fer à cheval, d'où l'appellation de la région du Golden Horseshoe. La région du Grand Toronto et de Hamilton (RGTH) comprend 2 municipalités à palier unique, soit Hamilton et Toronto, ainsi que 4 municipalités régionales et leurs 24 municipalités d'ordre inférieur. Quant à la région métropolitaine de Chicago, troisième en importance en termes de population aux États-Unis, elle s'étend sur toute la rive sud-ouest du lac Michigan. La région sous la juridiction de la commission régionale d'aménagement, la Chicago Metropolitain Agency for Planning (CMAP), comprend 7 comtés, englobant ainsi 284 municipalités. À la lecture du tableau de données, on remarque que les villes ont par ailleurs un territoire, une population et des densités de population semblables, quoique la région de Chicago est un peu plus grande, plus populeuse et un peu plus dense. La différence la plus importante a donc trait à la fragmentation institutionnelle, ou au nombre de municipalités. En effet, alors qu'on dénombre 26 municipalités et 4 municipalités régionales dans la région de Toronto, Chicago compte 284 municipalités et 7 comtés. Par ailleurs, la population de Toronto représente $40 \%$ de la population régionale, alors que celle de Chicago représente $31 \%$ de la population totale de la région. Tel qu'il sera discuté ultérieurement, cette plus grande fragmentation et décentralisation institutionnelle à Chicago n'est pas sans conséquence sur le processus de planification régionale.

En plus de leur ressemblance en matière de géographie, de démographie et d'importance en termes de transport, une agence de planification régionale a été créée dans chaque ville au cours des dernières années, témoignant de la volonté des autorités de la province, au Canada, et de l'État, aux États-Unis, d'apporter une solution au problème de la planification au niveau régional. C'est ainsi que Metrolinx fut créée par le gouvernement de l'Ontario en 2006 afin de coordonner la planification des transports dans la région de Toronto (en complément de l'adoption d'une stratégie de gestion de la croissance), et que la Chicago Metropolitain Agency for Planning (CMAP) fut instituée en 2005 par l'État de l'Illinois dans le but d'intégrer la planification des transports et de l'aménagement du territoire dans la région de Chicago. L'historique et la caractérisation de ces nouvelles institutions se retrouvent dans la section suivante.

\section{Politiques et pratiques}

Les prochains paragraphes font état des politiques, des pratiques et des processus en œuvre à Toronto et à Chicago. Ceux-ci sont catégorisés par niveau d'autorité, soit fédéral, provincial (Ontario) ou étatique (Illinois), métropolitain et municipal. 


\section{Toronto}

Gouvernement fédéral (Infrastructure Canada) L’implication du gouvernement fédéral canadien en matière de transport collectif se concrétise actuellement à travers la stratégie d'investissement en infrastructure Nouveau Plan Chantiers Canada (Infrastructure Canada, 2013), qui prévoit des investissements de l'ordre de 53 G \$ dans les infrastructures provinciales et municipales d'ici 2023. Dans l'ensemble, bien que le gouvernement fédéral ait considérablement accru les sommes attribuées aux infrastructures, au cours de la dernière décennie (Champagne, 2013), il n’y a pas d'enveloppe dédiée aux transports collectifs et on peut dire que l'intervention directe du niveau fédéral en matière d'affaires municipales a été historiquement épisodique (Young, 2013: 27).

À Toronto, le gouvernement fédéral est considéré par les répondants comme un partenaire financier de GO Transit, le système de trains de banlieue de la région, et de la Toronto Transit Commission, l'exploitant du réseau de métro, tramway et autobus de la région. Certains projets d'expansion et d'amélioration du réseau de transport sont effectivement bénéficiaires du Fonds canadien sur l'infrastructure stratégique, programme précurseur du Plan Chantiers Canada (Transport Canada, 2011). Cependant, les répondants n'ont identifié aucune politique du gouvernement fédéral explicitement liée au transport urbain et aucun programme relatif à l'aménagement du territoire. En effet, le gouvernement conservateur du premier ministre Stephen Harper n'intervient pas directement en la matière, reconnaissant explicitement que l'aménagement du territoire, ainsi que les affaires urbaines en général, sont de compétence provinciale et ne font pas partie de son mandat constitutionnel. En ce qui a trait au transport urbain, ces infrastructures font l'objet d'un financement important, mais ces contributions, motivées par des objectifs de compétitivité économique, ne sont pas encadrées par une politique ou un plan de transport fédéral. Cette position diffère des administrations précédentes des gouvernements libéraux des premiers ministres Jean Chrétien et Paul Martin, qui ont introduit le Nouveau pacte pour les villes et les communautés en 2005, auquel fut associé le transfert de la moitié des revenus de la taxe fédérale sur l'essence pour les projets d'infrastructures dites durables, incluant les transports collectifs (Young, 2013). Sous les gouvernements libéraux, chaque municipalité devait développer un plan de développement durable intégré démontrant comment les investissements locaux de la taxe fédérale sur l'essence contribueraient à la réduction des émissions de gaz à effet de serre et à la durabilité environnementale de la région. Ces plans sont toujours requis, mais les critères d'admissibilité aux fonds ont été réduits et élargis, de sorte qu'il n’y a plus cet accent sur la durabilité, comme c'était le cas sous les administrations précédentes (répondant 47). En outre, le gouvernement conservateur actuel a rendu permanent ce transfert de la taxe fédérale sur l'essence et l'a indexé à l'inflation (Infrastructure Canada, 2013). Comme on le verra plus loin, cette timide présence historique du gouvernement fédéral canadien dans le sujet qui nous occupe contraste avec une présence plus assurée du niveau fédéral étasunien.

Gouvernement provincial (Ontario) Le gouvernement provincial ontarien finance les projets d'expansion et d'amélioration du réseau de transport en commun, principalement exploité par la Toronto Transit Commission (TTC). De plus, le gouvernement libéral du premier ministre Dalton McGuinty a adopté, en 2005, deux législations importantes 
(introduites par le gouvernement conservateur précédent du premier ministre Mike Harris) ayant trait à l'aménagement du territoire: la Loi sur la ceinture de verdure et la Loi sur les zones de croissance. La Loi sur la ceinture de verdure et le Plan sur la ceinture de verdure qui y est associé ont institué une ceinture verte protégée d'environ 728000 ha de terres agricoles et écologiquement fragiles entourant la région de Toronto où l'urbanisation est interdite. Quant à la Loi sur les zones de croissance, elle permet au gouvernement provincial de désigner des centres de croissance métropolitains où doivent se trouver un plus grand nombre et une plus grande densité d'individus et d'emplois, afin de gérer la croissance démographique de la région, qui doit accueillir entre 100000 et 200000 nouveaux résidants par année (gouvernement de l'Ontario, 2006). Les municipalités doivent se conformer à la Loi sur les zones de croissance à travers la mise à jour de leur plan d'aménagement officiel, lequel fait l'objet d'une révision quinquennale. Les décisions municipales ayant trait à l'aménagement du territoire doivent aussi être effectuées conformément à la ceinture de verdure, au risque de faire l'objet d'un appel devant la Commission des affaires municipales de l'Ontario. De façon complémentaire à la stratégie de gestion de la croissance, le gouvernement provincial a aussi créé, en 2006, son agence métropolitaine de transport, Metrolinx, dont le mandat consiste à: 1) exploiter le système de trains de banlieue;2) mettre en œuvre le programme de système de trains légers de la région et de la navette ferroviaire rapide entre l'aéroport Pearson et le centre-ville de Toronto; et 3) coordonner la planification et l'intégration tarifaire des transports en commun pour toute la région, notamment au moyen d'une carte tarifaire électronique.

Agence métropolitaine (Metrolinx) Metrolinx est une entité quasi gouvernementale, soit une agence spécialisée qui relève du ministre provincial des Transports de l'Ontario. Il y a donc, à ce sujet, centralisation au niveau provincial. Lors de sa création, les membres du comité de direction de Metrolinx étaient recommandés par des municipalités, et le comité était ainsi composé majoritairement d'élus locaux. Par la suite, après l'adoption du plan régional de transport intitulé «Le grand projet», la composition du comité de direction fut modifiée pour remplacer les membres élus par des membres non élus, nommés par le gouvernement provincial sans la recommandation des municipalités, et représentant divers secteurs : financier, légal, transport, aménagement et communautaire, et ce, tout en assurant une représentativité géographique. Cette transition avait pour justification l'adoption du plan de sa mise en œuvre, de manière à susciter, en premier lieu, l'adhésion des élus locaux au plan régional de transport et, en second lieu, à faciliter sa mise en œuvre (répondant 47). Metrolinx contribue à l'intégration de la planification de l'aménagement du territoire et des transports de trois manières distinctes. D’abord, Le grand projet vise à relier les zones de croissance démographiques entre elles en bonifiant l'offre des transports collectifs rapides. De plus, Metrolinx utilise les données du Plan de croissance de la région élargie du Golden Horseshoe, adopté par la province, pour la prévision de la demande de transport. Finalement, l'agence a aussi mis sur pied des projets pilotes de centres de mobilité, comme la station de métro Kipling, où la station de train de banlieue, le métro, le réseau routier et l'aménagement du territoire sont mieux intégrés. Deux objectifs chiffrés de Metrolinx sont d'avoir $80 \%$ de la population vivant à moins de $2 \mathrm{~km}$ d'une station de transport collectif rapide d'ici 2031, ainsi que faire passer le temps de déplacement moyen dans la région d'environ 82 minutes à 77 minutes (répondant 18). 
Gouvernement municipal (Ville de Toronto) Le plan d'aménagement officiel de Toronto dirige la croissance démographique vers les secteurs déjà desservis par les transports en commun, en conformité avec la législation provinciale. À la suite d'une consultation publique, la Ville de Toronto a défini le centre-ville et quatre centres urbains comme étant les importants centres de croissance qui seront redéveloppés et intensifiés, alors qu'environ $75 \%$ du territoire, constitué de quartiers résidentiels et de parcs, seront essentiellement gardés intacts. Les centres de croissance sont constitués d'environ $160 \mathrm{~km}$ d'avenues comprenant des plazzas commerciales vieillissantes, de petites zones de stationnement, ainsi que d'autres bâtiments de piètre qualité âgés d'une cinquantaine d'années. Ces zones déjà bien desservies par les transports collectifs sont prêtes à être rebâties et intensifiées. En permettant la construction d'un immeuble de six ou huit étages avec commerce au premier niveau, par exemple, les autorités municipales ont comme objectif une meilleure utilisation du territoire. La Ville de Toronto n'a pas d'objectif chiffré de croissance de la part modale des transports collectifs; elle vise plutôt à mettre en œuvre des politiques qui augmentent l'utilisation des moyens de transport autres que l'automobile, comme des plans favorisant l'utilisation des transports durables, le réseau cyclable et les déplacements à pied, et qui supportent le développement d'une région urbaine où «il fait bon vivre» avec des quartiers disposant de services à proximité (répondant 13).

\section{Chicago}

Gouvernement fédéral (Department of Transportation - DOT, Department of Housing and Urban Development - HUD \& Environmental Protection Agency - EPA) Le gouvernement fédéral américain est un important partenaire financier des projets de transport sélectionnés par les agences métropolitaines de transport (Metropolitan Planning Organizations - MPO). En fait, le DOT requiert l'existence des MPO dans les régions métropolitaines de plus de 50000 habitants, depuis l'adoption de l'Interstate Highway Act de 1973, ainsi que l'élaboration par elles d'un programme financier d'amélioration des transports (Transportation Improvement Program) devant répondre à plusieurs critères afin de pouvoir bénéficier des subventions fédérales en transport. Une nouvelle initiative du gouvernement fédéral américain, le Sustainable Communities Regional Planning Grant Program, pilotée par le DOT en collaboration avec le HUD et l'EPA, facilite la mise en œuvre des plans métropolitains de transport et d'aménagement en finançant des projets locaux faisant appel à la collaboration entre plusieurs partenaires et à l'inclusion de plusieurs aspects de l'aménagement (HUD, 2011). À Chicago, cela s'est traduit par l'octroi, en 2010, d'une subvention de 4,25 M \$ finançant le programme d'assistance technique local de l'agence de planification régionale (qui agit aussi à titre de MPO), mettant ainsi à la disposition des municipalités 10 urbanistes pour actualiser leur plan de zonage et réaliser des projets respectant les principes du plan de transport et d’aménagement métropolitain.

Gouvernement de l'État (Illinois) Le gouvernement de l'État de l'Illinois est considéré comme un partenaire financier, collectant la taxe de vente dont une partie est redistribuée par l'autorité régionale des transports collectifs (Regional Transit Authority) aux trois opérateurs de transport métropolitain, soit: 1) CTA, la Chicago Transit Authority, qui regroupe les services d'autobus et de métro pour la ville de Chicago et 40 banlieues immédiates;2) Metra, le service de trains de banlieue; et 3) Pace, le service d'autobus et de transport adapté fonctionnant dans quelque 283 villes 
ou municipalités en banlieue de Chicago. Nos répondants n’ont pointé aucune politique spécifique dédiée à la réduction de l'«auto-solo» et aucun programme ou loi concernant l'aménagement du territoire géré par l'État de l'Illinois, ce qui contraste avec la province de l'Ontario, plus interventionniste. Cependant, c'est le gouvernement de l'État qui a aboli la commission des transports (Chicago Bureau of Transportation Study - CATS) et la commission d'aménagement du territoire (Northeastern Illinois Planning Commission - NIPC) pour créer, en 2005, la Chicago Metropolitain Agency for Planning (CMAP), dont le mandat est d'intégrer la planification des transports et l'aménagement du territoire et qui agit aussi en tant que MPO pour la région de Chicago. Cette initiative fut instiguée par une campagne de lobbyisme de deux ans menée par une organisation régionale sans but lucratif représentant la communauté des affaires, Metropolis Strategies, laquelle orchestra une campagne médiatique, forgea une coalition des deux partis siégeant à l'Assemblée législative, trouva des porteurs de dossiers et rédigea le projet de loi qui fut adopté à l'unanimité par les membres de l'Assemblée générale de l'État de l'Illinois (répondant 31).

Agence métropolitaine (Chicago Metropolitan Agency for Planning - CMAP) CMAP est dirigée par deux comités de direction: le comité de CMAP (CMAP Board) qui est responsable essentiellement des plans et politiques d'intégration des transports et d'aménagement du territoire, ainsi que le comité politique du MPO (MPO Policy Committee), responsable de la distribution d'une partie des fonds fédéraux en transport. Le comité de direction de CMAP est composé à moitié d'élus de la région et à moitié de gens de la communauté des affaires et de la société civile. La distribution des membres est proportionnelle au poids démographique des comtés et municipalités de la région. Ainsi, cinq membres sont nommés par la Ville de Chicago, cinq représentent le comté de Cook (Chicago et ses banlieues immédiates), et cinq représentent les comtés de la couronne (banlieues éloignées). De plus, une proposition doit obtenir une forte majorité pour être adoptée, soit 12 votes sur 15. Cette composition ainsi que ce mode de fonctionnement ont été motivés par les désirs de concertation et de consensus régional ayant mené à la fondation de l'organisation. Quant au comité politique du MPO, il est régi conformément à la législation fédérale et composé de représentants de chaque palier de gouvernement, d'autorité régionale et d'agence de transport, comme cela était le cas avant la fusion des deux agences régionales, sous CATS (CMAP, 2014). Dans les faits, le comité de CMAP, responsable des plans et des politiques, est contrôlé par les acteurs locaux, alors que le comité politique du MPO, responsable du financement des projets de transport, est contrôlé par le ministre des Transports (répondant 25).

Le plan régional de transport et d'aménagement du territoire de CMAP, Go to 2040, fut adopté à l'unanimité en 2009 par les membres du comité de CMAP à la suite d'un long processus de consultation ayant suscité l'attention des autres MPO aux ÉtatsUnis, notamment pour le caractère exhaustif du plan et pour l'outil interactif Web novateur utilisé lors de la consultation publique. Bien que CMAP n’ait aucun pouvoir sur le zonage et l'aménagement du territoire, qui demeurent des prérogatives des gouvernements municipaux, la mise en œuvre de Go to 2040 au niveau local est facilitée par le programme local d'assistance technique financé par le gouvernement fédéral (tel que mentionné précédemment). Ainsi, le Local Technical Assistance Program met 10 urbanistes au service des municipalités afin d'en actualiser les plans de zonage et afin de concevoir ou de réaliser conjointement des projets en lien avec Go to 2040. 
Gouvernement municipal (Ville de Chicago) Les municipalités de la région métropolitaine de Chicago ne sont soumises à aucun critère de densité ou de gestion de la croissance sur leur territoire. Cependant, la Ville de Chicago a mis de l'avant plusieurs initiatives visant à susciter le redéveloppement autour des corridors de transport en commun. Ainsi, la Ville et la CTA ont conçu le Transit Friendly Development Guide à l'attention des conseillers municipaux et des promoteurs privés, afin d'encourager un développement propice au transport collectif aux alentours des stations de métro et de déceler des occasions de partenariat ou de redéveloppement de propriétés appartenant à la Ville ou à la CTA (Department of Zoning and Land Use Planning \& Chicago Transit Authority, 2009). Ce guide regroupe 144 stations de métro ou de train selon 7 typologies auxquelles sont associés des objectifs de densité, d'activité, de mixité et de zonage, selon la trame urbaine environnante. Cependant, ce guide n'a qu'une portée informative et incitative. Aussi, plus récemment, la Ville a adopté un décret pour le développement orienté vers le transport en commun (TOD Ordinance), qui accroît les incitatifs pour le développement résidentiel et commercial de qualité près des stations de métro en: 1) diminuant le nombre de stationnements obligatoires;2) augmentant le coefficient d'occupation des sols ; 3) augmentant la hauteur permise des édifices ; et 4) diminuant la superficie minimum du lot par unité (City of Chicago, 2013). Ce décret a pour objectif d'éliminer les barrières à la construction et au redéveloppement de bâtiments plus denses et orientés vers les transports collectifs à proximité des stations de métro.

\section{Discussion et conclusion}

L'objectif de cet article était de documenter les réformes institutionnelles visant à mieux intégrer la planification des transports et de l'aménagement du territoire en mettant en exergue les dimensions verticales et horizontales de la gouvernance régionale. À Toronto, l'intégration de l'aménagement du territoire et des transports au niveau métropolitain se fait à travers les plans officiels municipaux qui dirigent la croissance démographique dans certains secteurs, en conformité avec la législation provinciale, ainsi qu'à travers le mandat de Metrolinx, qui relie les zones de croissance entre elles par la bonification du réseau de transport collectif. Au final, en comparaison avec Chicago, on peut dire que la composante hiérarchique et coercitive est plus présente à Toronto. À Chicago, l'intégration se fait principalement de manière décentralisée à travers le programme d'assistance à la planification locale de CMAP pour les municipalités disposant de moins de ressources, financé par le gouvernement fédéral, mais aussi grâce à l'initiative volontaire de la Ville de Chicago, dans le cas du décret municipal pour le développement orienté vers le transport en commun. En outre, on ne peut passer sous silence l'importance de l'initiative de Metropolis Strategies, une organisation du milieu des affaires, qui a suscité l'intégration de la planification des transports et de l'aménagement du territoire à travers la création de CMAP. Cette mise à l'agenda constitue un indicateur additionnel de la présence d'une gouvernance horizontale à Chicago, mais aussi de la manière dont peut se réaliser la séquence des dynamiques horizontales et verticales. On peut certainement dire que, dans le cas du processus de création de CMAP, la gouvernance, dans ses dimensions décentralisées et horizontales, a été efficace dans son influence d'un niveau de gouvernement d’ordre supérieur. Cependant, un autre aspect de la décentralisation et de l'horizontalité des processus à Chicago semble nuire à la mise en œuvre de l'intégration efficace entre les dimensions transport et aménagement du territoire, à l'échelle régionale. En effet, la réforme institutionnelle 
n’a pas fondamentalement modifié les exigences ou le mode décisionnel relatifs au financement des projets de transport et à l'utilisation du sol, lesquels sont pris dans des instances contrôlées par le gouvernement de l'État (comité politique du MPO) et les gouvernements locaux (cabinets des maires ou conseils exécutifs), respectivement, comme cela était le cas avant la réforme.

Ce dernier point nous réfère au design institutionnel des nouvelles agences de planification métropolitaines et à l'importance des mandats et des pouvoirs de mise en œuvre qui leur sont conférés par les niveaux supérieurs de gouvernement. Alors que Metrolinx, une agence du gouvernement de l'Ontario, a un mandat de coordination, de financement et d'exploitation des transports, CMAP, contrôlée par les acteurs métropolitains de la région de Chicago, a un mandat de planification à la fois des transports et de l'aménagement du territoire, mais dispose d'un financement et d'une capacité de mise en œuvre plus limités (le financement étant contrôlé par le comité du MPO, la coordination des opérateurs en transports, par la RTA, et l'utilisation du sol, par les municipalités). Le fait que Metrolinx, une corporation de la Couronne, soit à la fois propriétaire et exploitante de transports en commun semble motiver le gouvernement provincial à investir des sommes plus importantes et lui permettre de les amortir sur une plus longue période. Cela rendrait aussi possible une meilleure reddition de compte, puisque les fonds passent à une agence relevant du gouvernement provincial, au lieu d'être transférés à une municipalité ou à une agence de transport indépendante. Tel que décrit par un répondant de la région de Toronto, ce changement important en termes de gouvernance, qui ébranle le statu quo, n'est pas sans froisser les autorités traditionnelles (répondant 24). Quant à CMAP, elle ne dispose pas d'une capacité financière suffisante et de l'autorité sur le zonage et l'aménagement du territoire qui lui permettraient de mettre en œuvre elle-même son plan Go to 2040 au niveau régional. Elle dépend donc entièrement du programme d'assistance technique local et de la coopération volontaire des municipalités qui, au-delà de Chicago et de ses banlieues immédiates, perçoivent CMAP comme une menace au développement urbain et à l'accroissement de leur assiette fiscale.

Ces résultats démontrent l'importance de la gouvernance verticale pour promouvoir une vision métropolitaine, comme dans le cas de l'intervention de la province de l'Ontario dans la région de Toronto. La création de CMAP et la campagne de lobbyisme orchestrée par Metropolis Strategies démontrent aussi l'importance de la gouvernance horizontale et du capital civique au niveau métropolitain pour provoquer des changements aux paliers supérieurs, en l'absence de volonté politique. Par contre, le cas de Chicago illustre aussi les limites de l'approche volontaire et décentralisée, et le fait qu'une plus grande fragmentation constitue un frein à la mise en œuvre de plans métropolitains d'aménagement. En effet, même si l'État de l'Illinois décidait d'adopter une politique de gestion de la croissance, comme celle qui est mise en application en Ontario ou ailleurs aux États-Unis, il faudrait que cette politique soit mise en application dans plus de 280 municipalités, et l'exercice de conformité et de reddition de compte serait d'autant plus laborieux.

Sur la base de nos observations, il est difficile d'imaginer comment une région métropolitaine pourrait relever le défi de l'intégration de l'aménagement du territoire et des transports sans la présence de certains éléments plus verticaux de la gouvernance et sans donner aux entités créées la capacité d’accomplir leur mandat. Or, le processus de 
création d'une nouvelle institution est le théâtre des jeux de pouvoir entre acteurs pour le contrôle des ressources, duquel résulte un compromis politique plus ou moins favorable à l'atteinte des objectifs. En ce sens, le cas de l'adoption de la stratégie de croissance dans le sud de l'Ontario constitue un véritable tour de force de la part du gouvernement provincial pour imposer une intensification du territoire, ainsi qu'une protection du territoire agricole et d'écosystèmes sensibles. Quant à Metrolinx, le changement de la composition du comité de direction et le remplacement d'un conseil contrôlé par les municipalités par un conseil contrôlé par le gouvernement provincial représentent un bris de consensus régional dont les conséquences restent à être évaluées. Dans le cas de Chicago, le compromis entourant la création de CMAP montre à quel point le home rule américain, profondément ancré dans cet État du Midwest, complexifie la mise en œuvre coordonnée d'une stratégie régionale d'utilisation du territoire et soulève des questions quant à l'étendue de l'impact de la composition des instances attribuant le financement des projets de transport sur les types de projets financés.

Les révisions du plan de croissance et du Grand projet, dans la région de Toronto, et de Go to 2040, dans la région de Chicago, prévues dans leur statut en 2015-2016 et 2014 respectivement, permettront d'évaluer leur mise en œuvre au niveau local. En outre, nos travaux tentent de mettre en exergue plus précisément la manière dont certains éléments structurels, comme la sélection et la composition des comités de direction, l'étendue des pouvoirs et des ressources et les obligations légales, constituent réellement un frein ou un moteur pour la planification régionale. Nos travaux tentent également d'évaluer les impacts de ces éléments sur le financement des projets de transport et les décisions relatives à l'utilisation du sol.

Sans préjuger du succès futur de l'intégration des transports et de l'aménagement du territoire à Toronto et à Chicago, nous pouvons déjà conclure sur certains éléments institutionnels. Nous avions amorcé cette étude en nous interrogeant sur l'utilisation d'éléments verticaux et top down afin de surmonter les défis de la gouvernance multiniveau à l'échelle métropolitaine. Au regard de notre étude institutionnelle, nous concluons qu'il ne faut pas exclure, dans nos perceptions de ce qu'est la gouvernance, le fait que la dimension verticale, non seulement existe, mais constitue un passage obligé à certaines étapes cruciales de la réalisation des politiques publiques en matière d'intégration de la planification régionale des transports et de l'aménagement du territoire. 


\section{Bibliographie}

ALEXANDER, Lisa T. (2010) The promise and perils of 'new regionalist' approaches to sustainable communities. Fordham Urban Law Journal, vol. 38, nº3, p. 627-674.

BLACK, William R. (2010) Sustainable transportation: Problems and solutions. New York, Guilford Press.

BOURDAGES, Jade et CHAMPAGNE, Éric (2012) Penser la mobilité durable au-delà de la planification traditionnelle du transport. VertigO, Hors-série, vol.11, p. 1-22.

BRENNER, Neil (2002) Decoding the newest 'metropolitan regionalism' in the USA: A critical overview. Cities, vol. 19, no 1 , p. 3-21.

CALTHORPE, Peter et FULTON, William (2001) The regional city: Planning for the end of sprawl. Washington, D.C., Island Press.

CERVERO, Robert et DUNCAN, Michael (2006) Which reduces vehicle travel more: Job-housing balance or retail-housing mixing? Journal of the American Planning Association, vol. 72, nº 4, p. 475-490.

CHAMPAGNE, Eric (2013) Les programmes d'infrastructures municipales du gouvernement fédéral: une analyse de la gouvernance multiniveau au Canada. Télescope, vol. 19, nº 1, p. 43-61.

CITY OF CHICAGO (2013) Final substitute ordinance - Chicago zoning ordinance [En ligne]. http://www.metroplanning. org/uploads/cms/documents/tod_ ordinance.pdf

CITY OF CHICAGO et CHICAGO TRANSIT AUTHORITY (2009) Chicago transit friendly development guide [En ligne]. http://www.cityofchicago.org/city/ en/depts/dcd/supp_info/transit_ friendlydevelopmentguide.html

CMAP (Chicago Metropolitan Agency for Planning) (2014) CMAP Board [En ligne]. http://www.cmap.illinois.gov/about/ involvement/committees/cmap-board
DATA MANAGEMENT GROUP (2011) City of Toronto. Transportation Tomorrow, 2011 Survey Area Summary [En ligne]. http://dmg.utoronto.ca/pdf/tts/2011/ travel_summaries_for_the_gtha/Toronto/ City_of_Toronto_summary.pdf

DEAL, Brian, KIM, Jae Hong et CHAKRABORTY, Arnab (2009) Growth management and sustainable transport: Do growth management policies promote transit use? Journal of Public Transportation, vol. 12, $\mathrm{n}^{\circ} 4$, p. 21-40.

DEPARTMENT OF ZONING AND LAND USE PLANNING \& CHICAGO TRANSIT AUTHORITY (2009) Transit friendly development guide. City of Chicago [En ligne]. http://www.cityofchicago.org/ city/en/depts/dcd/supp_info/transit_ friendlydevelopmentguide.html

DIVAY, Gérard et PAQUIN, Stéphane (2013) L'administration publique dans la gouvernance multiniveau infranationale: état de la question et perspectives. Télescope, vol. 19, nº 1, p. 1-24.

FORSYTH, Ann, OAKES, J. Michael et SCHMITZ, Kathryn H. (2007) Does residential density increase walking and other physical activity? Urban Studies, vol. 44, nº 4, p. 679-697.

FLYVBJERG, Bent (2006) Five misunderstandings about case-study research. Qualitative Inquiry, vol. 12, p. 219-245.

FREEMARK, Yonah (2009) Transit mode share trends looking steady: Rail appears to encourage non-automobile commute. TheTransportPolitic.com [En ligne]. http:// www.thetransportpolitic.com/2010/10/13/ transit-mode-share-trends-looking-steadyrail-appears-to-encourage-non-automobilecommutes/

GIULIANO, Genevieve (1995) The weakening transportation-land use connection. Access, vol.6, p. 3-11. 
HAMILTON, David, HOKKANEN, Laurie et WOOD, Curtis (2008) Are we still stuck in traffic? Transportation in metropolitan areas. Dans David Hamilton et Patricia Atkins (dir.) Urban and regional policies for metropolitan livability. Londres, M.E. Sharpe Publishers, p. 266-295.

HANDY, Susan, CAO, Xinyu et MOKHTARIAN, Patricia (2005) Correlation or causality between the built environment and travel behavior? Evidence from Northern California. Transportation Research Part D, vol. 10.

HIBBING, John R. et THIESSE-MOSS, Elizabeth (2001) What is it about government that Americans dislike. Cambridge, Cambridge University Press.

HORAK, Martin (2012a) Multilevel governance in Toronto: Success and failure in Canada's largest city. Dans Martin Horak et Robert Young (dir.) Sites of governance. Multilevel governance and policy making in Canada's big cities. Montréal et Kingston, McGillQueen's University Press, p. 228-262.

HORAK, Martin (2012b) Conclusion: Understanding multilevel governance in Canada's. Dans Martin Horak et Robert Young (dir.) Sites of governance. Multilevel governance and policy making in Canada's big cities. Montréal et Kingston, McGillQueen's University Press. p. 339-370.

HORAK, Martin et YOUNG, Robert (dir.) (2012) Sites of governance. Multilevel governance and policy making in Canada's big cities. Montréal et Kingston, McGillQueen's University Press.

HUD (U.S. Department of Housing and Urban Development) (2011) HUD FY2011 Sustainable Communities Grantees [En ligne]. http://portal.hud.gov/ hudportal/HUD?src=/program_offices / sustainable_housing_communities / sustainable_communities_regional_ planning_grants

INFRASTRUCTURE CANADA (2013) Nouveau Plan Chantiers Canada: le plus vaste et le plus long plan fédéral d'infrastructure de l'histoire canadienne [En ligne]. http:// www.infrastructure.gc.ca/plan/nbcpnpcc-fra.html
JOHNSTON, Robert (2004) The urban transportation planning process. Dans Susan Hanson et Genevieve Giuliano (dir.) The geography of urban transportation. Third Edition. New York, Guilford Press, p. 115-140.

KLIJN, Erik-Hans et KOPPENJAN, Joop (2012) Governance network theory: Past, present and future. Policy \& Politics, vol. 40, n ${ }^{\circ}$, p. 587-606.

LITMAN, Todd (2013) The new transportation planning paradigm. ITE Journal, Juin 2013, p. 20-28.

MARGERUM, Richard, BRODY, Susan, PARKER, Robert et MCEWEN, Gail (2011) Regional transportation and land use decision making in metropolitan regions: Findings from four case studies. National Policy Consensus Center [En ligne]. http:// www.policyconsensus.org/publications/ reports/trans_landuse.pdf

NELLES, Jen (2013) Cooperation and capacity? Exploring the sources and limits of city region governance partnerships. International Journal of Urban and Regional Research, vol.37, nº4, p. 1349-1367.

NEUMAN, Michael (2005) The compact city fallacy. Journal of Planning Education and Research, vol.25, n 11 , p. 11-26.

NORTON, Alan (1994) International handbook of local and regional government: A comparative analysis of advanced democracies. Chenltenham, Edward Elgar.

PIERRE, Jon et PETERS, Guy (2012) Urban governance. Dans Karen Mossberger, Karen, Susan E. Clarke et Peter John (dir.) The Oxford handbook of urban politics. Oxford University Press.

PORTER, Douglas R. (1992) State and regional initiatives for managing development: Policy issues and practical concerns. Washington, DC, Urban Land Institute.

RHODES, Roderick A.W. (1996) The new governance: Governing without government. Political Studies, vol.44, p. 652-667.

RIGGS, Fred W. (1998) Public administration in America: Why our uniqueness in exceptional and important. Public Administration Review, vol. 58, $\mathrm{n}^{\circ} 1$, p. 22-31.

Intégration des transports et de l'aménagement du territoire au niveau métropolitain à Toronto et à Chicago 
STATISTIQUE CANADA (2012) Toronto, Ontario (Code 3520) et Ontario (Code 35) (table). Census Profile. 2011 Census. Statistics Canada Catalogue no. 98-316XWE. Ottawa [En ligne]. http://www12. statcan.gc.ca/census-recensement/2011/ dp-pd/prof/index.cfm?Lang=E

TRANSPORT CANADA (2011) Current Projects. Surface Infrastructure [En ligne]. https://www.tc.gc.ca/eng/programs/ surface-transit-projects-221.htm

TRANSPORTATION RESEARCH BOARD (2009) Driving and the built environment: The effects of compact development on motorized travel, energy use, and $\mathrm{CO}_{2}$ emissions. Special Report 298. Washington, DC, National Academy Press.

WEIR, Margaret (2000) Coalition building for regionalism. Dans Bruce Katz (dir.) Reflections on regionalism. Washington, D.C, Brookings Institutions Press, p. 127-153.

WEIR, Margaret, RONGERUDE, Jane et ANSELL, Christopher (2009) Collaboration is not enough: Virtuous cycles of reform in transportation policy. Urban Affairs Review, vol.44, n 4, p. 455-489.

WHEELER, Stephen (2002) The new regionalism: Key characteristics of an emerging movement. Journal of the American Planning Association, vol.68, no3, p. 267-278.

YIN, Robert (2003) Case study research: Design and methods. Third edition. Sage.

YOUNG, Robert (2012) Introduction: Multilevel governance and its central research questions in Canadian cities. Dans Martin Horak et Robert Young (dir.) Sites of governance. Multilevel governance and policy making in Canada's big cities. Montréal et Kingston, McGill-Queen's University Press, p. 3-25.

YOUNG, Robert (2013) La gouvernance multiniveau et les politiques publiques au sein des municipalités du Canada: reddition de comptes et efficacité. Télescope, vol. 19, no 1, p. 25-42. 